\title{
The expression of B7-H1 and B7-H4 molecules on immature myeloid and lymphoid dendritic cells in cord blood of healthy neonates
}

\author{
Kludka-Sternik $\mathbf{M}^{\mathbf{1}}$, Serafin $\mathbf{A}^{\mathbf{1}}$, Darmochwal-Kolarz $\mathbf{D}^{\mathbf{2}}$, Radej $\mathbf{S}^{\mathbf{1}}$, Rolinski $\mathbf{J}^{\mathbf{1}}$, \\ Leszczynska-Gorzelak $\mathrm{B}^{2}$, Oleszczuk $\mathbf{J}^{2}$
}

${ }^{1}$ Department of Clinical Immunology, Medical University of Lublin, Poland

${ }^{2}$ Department of Obstetrics and Perinatology, Medical University of Lublin, Poland

\begin{abstract}
The aim of our study was to estimate both B7-H1 and B7-H4 molecules on immature myeloid and lymphoid dendritic cells in umbilical cord blood of healthy neonates in comparison with peripheral blood of healthy adults. Thirty nine healthy full-term neonates from physiological single pregnancies and 27 healthy adults were included in the study. The expression of B7-H1 and B7-H4 was revealed using the immunofluorescence method. Statistical analysis was performed using a non-parametric test (Mann-Whitney U-Test). The percentages of BDCA- $1^{+}$dendritic cells with B7-H1 and B7-H4 expressions were significantly higher in peripheral blood of healthy adults $(\mathrm{p}<0.00003)$. It was either observed that the percentage of BDCA- $2^{+}$dendritic cells with the expression of B7-H4 molecules was significantly higher in peripheral blood of healthy adults in comparison with umbilical cord blood $(\mathrm{p}<0.02)$. Decreased percentages of dendritic cells and co-stimulatory molecules indicate that neonates have immature immune system. Depletion of co-stimulatory B7-H1 and B7-H4 molecules enable appropriate development of immune response.
\end{abstract}

Key words: B7-H1, B7-H4, BDCA-1+, BDCA-2+, cord blood, neonates.

\section{Introduction}

The immune system of neonate is completely different from that from the adults. In cord blood, dendritic cells (DCs) dominate that mean that neonatal immunological response is still immature. The immunological changes as well as maturation are present before and just after birth. The birth gives rise to appropriate response, where DCs contain the first line of the defense. They are specialized antigen-presenting cells (APCs). We can distinguish two types of DC: myieloid $(\mathrm{mDC})$ and lymphoid dendritic cells (pDC). They are mainly responsible for presenting antigens to naive lymphocyte $\mathrm{T}$ cells. It is said that are also able to induce immunological tolerance [1-4].

The antigen B7-H1 belongs to the newly discovered B7 immunomodulatory proteins family and it is a costimulatory protein with properties of inhibiting T-cell

Correspondence: Kludka-Sternik M, Dept. of Clinical

Immunology, Medical University of Lublin, Poland; 20-950

Lublin, Jaczewskiego Str, 8, Poland; tel./fax.: (+4881) 7187315 ,

e-mail: magdalenakludka@go2.pl response. It ligates CD28 antigen family and either interacts with antigen called programmed death-1 (PD1 ), thus the high expression of this molecule may transmit an inhibitory signal reducing T-cells responses. The molecules - B7 and CD28 are the part of immunoglobulin (Ig) superfamily of type I transmembrane proteins [5]. The molecule - B7 may interact with both CD28 as well as with CTLA-4 inhibitory receptors that are present on antigen-presenting cells [6].

The molecule B7-H4 is also B7 membrane family that down-regulates $\mathrm{T}$ cells responses. It is able to inhibit the proliferation of $\mathrm{T}$ cells, reduces Th1 and Th2 cytokine production and cell cycle progression ligating to its receptor BTLA on T lymphocytes [7]. There are known some cytokines which regulate the expression of B7-H4 molecule. Interleukin-10 (IL-10) up-regulates whereas GM-CSF or IL-4 reduce its expression. It was either shown that overexpression of this molecule is present on ovarian cancer tissues and thus it may be used as a marker of tumors [8-10].

The aim of our study was to investigate if there is an expression of co-stimulatory molecules both B7-H1 
and $\mathrm{B} 7-\mathrm{H} 4$ on immature myeloid and lymphoid dendritic cells in umbilical cord blood of healthy neonates in comparison with peripheral blood of healthy adults.

\section{Material and methods}

Patients. The study population included 39 full-term newborns born from physiological pregnancies. The deliveries took place in the Department of Obstetrics and Perinatology, Medical University of Lublin. Blood samples were collected from umbilical cord throughout labor. The control group comprised of 27 healthy adults.

Cell preparation. Twenty milliliters of blood were taken from the study and control groups and collected in heparinized tubes (Polfa, Warsaw, Poland). Mononuclear cells (MC) were separated on lymphocyte separation medium - Gradisol L (Aqua Medica, Poland) and centrifuged for 20 minutes at $700 \times \mathrm{g}$. Every $10^{7}$ of MC were incubated with $10 \mu \mathrm{l}$ of FcR - Blocking Reagent (Miltenyi Biotec, Germany) for 5 minutes in 22 degrees of Celsius to avoid non-specific binding. Then, cells were labeled with monoclonal antibodies. Five $\mu \mathrm{l}$ of appropriate antibodies against following antigens: BDCA-1, BDCA-2, as well as B7-H1, B7-H4 were added to each sample. After incubation, cells were centrifuged, washed for 5 minutes at 700 $\times \mathrm{g}$ in 4 degrees of Celsius. Next, cells were analyzed in the flow cytometer. The following combinations of antibodies were used for myeloid dendritic cells: anti - BDCA-1 (CD1c) FITC monoclonal antibody (Miltenyi Biotec, Germany) and anti - CD19 CyChrome monoclonal antibody (Pharmingen, USA); anti - BDCA-1 (CD1c) FITC monoclonal antibody (Miltenyi Biotec, Germany), anti-B7-H1 PE monoclonal antibody (AbD Serotec, USA) and anti-CD19 CyChrome monoclonal antibody (Pharmingen, USA); anti - BDCA1 (CD1c) FITC monoclonal antibody (Miltenyi Biotec, Germany), anti-B7-H4 PE (AbD Serotec, USA) and anti-CD19 CyChrome monoclonal antibody (Pharmingen, USA).

The following combinations of antibodies were used for lymphoid dendritic cells: anti - BDCA-2 (Miltenyi Biotec, Germany); anti - BDCA-2 FITC (Miltenyi Biotec, Germany) and anti- B7-H1 monoclonal antibody PE (AbD Serotec, USA); anti - BDCA-2 FITC monoclonal antibody (Miltenyi Biotec, Germany) and antiB7-H4 PE monoclonal antibody (AbD Serotec, USA).

The cell phenotype characterization was performed using a FACSCalibur equipped with 488-nm argon laser (Becton Dickinson, USA) and analyzed with CellQuest Software (Becton Dickinson, USA). We collected 300000 of events in total. Cell debris and dead cells were excluded from the analysis based on scatter signal.

Ethical issues. The study design was accepted by the local Ethics Committee. Informed consent from the delivering patients and healthy adults for blood sampling was obtained.

Statistic analysis. Statistical differences between groups were estimated using a standard non-parametric test (Mann-Whitney U-test). The results were presented as median and interquartile ranges. Differences at $\mathrm{p}<0.05$ were considered as statistically significant. Statistica 7.1 PL software was applied to statistical analysis.

\section{Results}

The expressions of B7H1 and B7H4 co-stimulatory molecules were present on immature myeloid BDCA$1+$ and lymphoid BDCA-2+ dendritic cells. It was observed that the percentages of BDCA-1+ dendritic cells with $\mathrm{B} 7-\mathrm{H} 1$ and $\mathrm{B} 7-\mathrm{H} 4$ molecule expressions were significantly higher in peripheral blood of healthy adults when compared to neonates $(p<0.0001)$. Moreover, the percentages of BDCA- $2^{+}$dendritic cells with the expressions of $\mathrm{B} 7-\mathrm{H} 4$ molecule were significantly higher in peripheral blood of healthy adults in comparison with umbilical cord blood $(\mathrm{p}<0.02)$. Furthermore, we noticed that the percentages of both group of DCs cells were lower in cord blood when compared to peripheral blood of healthy adults. The results are presented in the Figs 1, 2, 3, 4 and 5.

\section{Discussion}

It is well known that although neonatal immune system is developed, it is still immature just after birth. When fetus grows, many changes have their onset at that time and then cells become more specialized. In this study using appropriate gating strategy we identified $\mathrm{BDCA}^{-}{ }^{+}$and $\mathrm{BDCA}-2^{+}$subsets of dendritic cells. It may indicate that neonatal immune system is still under the control of maternal hormones. It was described that hormones are able to influence on DC [11-14]. There are works describing that progesterone is one of the agents that naturally inhibit cell-mediated immunity. It is capable to modulate a differentiation, maturation and function of DCs. Progesterone inhibits immune responses by producing more immature bone marrow (BM) [11]. Furthermore, progesterone can be the inducer of Th2 response. Because of the high concentration of these hormones in the fetal-maternal interface, fetus is tolerated by maternal organism. After birth, a neonate is still under the maternal hormone control, the concentrations of the hormones are still elevated in umbilical cord blood. This situation may explain lower levels of BDCA- $1^{+}$and BDCA-2 ${ }^{+}$ in cord blood of neonates in comparison with peripheral blood of adults. Prostaglandin - PGE2 present in the maternal-fetal interface can induce the production of DCs. It is well known that PGE2 polarizes the maturation of $\mathrm{mDC}$ into Th2-promoting DCs $[15,16]$. There were also studies where it was shown that the levels of cortisol correlated with DCs counts.

Szabolcs et al. demonstrated in their study that there was a difference in absolute number of pDCs in cord blood in comparison with peripheral one. They observed lower concentrations of pDCs in volunteer blood in comparison with cord blood. It was also noticed lower number of $\mathrm{mDCs}$ in healthy adults. Moreover, in cord blood and adult peripheral blood there were almost twice as many mDCs than pDCs. However, we did not observe this kind of correlation [17].

There is a predominance of Th2 type immunity in a neonatal immune system. There are works where it was described that there are higher numbers of $\mathrm{pDC}$ in umbilical cord blood. It was either marked that neonatal immune response is impair and therefore the abili- 

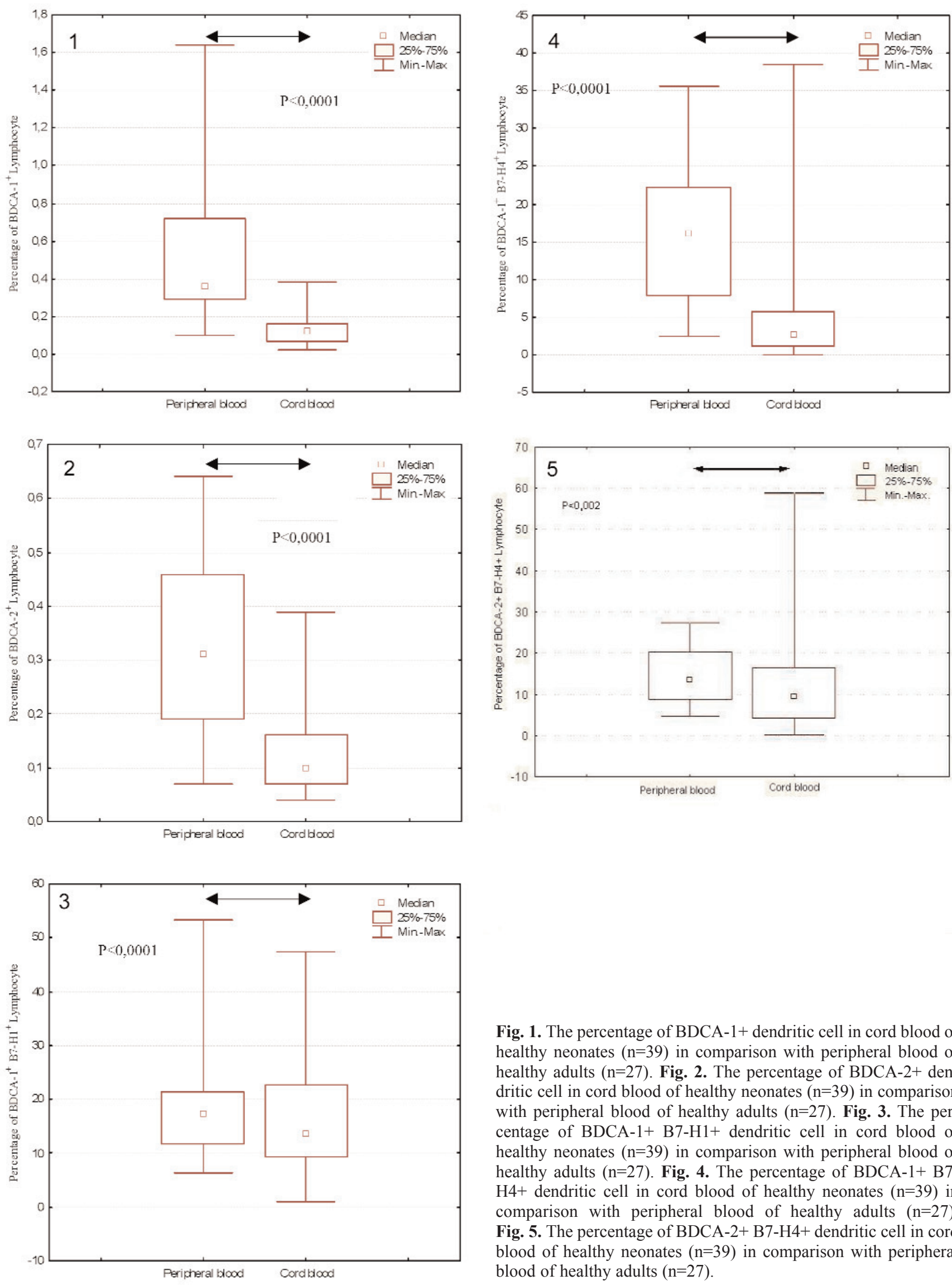

Fig. 1. The percentage of BDCA-1+ dendritic cell in cord blood of healthy neonates $(n=39)$ in comparison with peripheral blood of healthy adults $(n=27)$. Fig. 2. The percentage of BDCA-2+ dendritic cell in cord blood of healthy neonates $(n=39)$ in comparison with peripheral blood of healthy adults $(n=27)$. Fig. 3. The percentage of BDCA-1+ B7-H1+ dendritic cell in cord blood of healthy neonates $(n=39)$ in comparison with peripheral blood of healthy adults $(n=27)$. Fig. 4. The percentage of BDCA-1+ B7$\mathrm{H} 4+$ dendritic cell in cord blood of healthy neonates $(n=39)$ in comparison with peripheral blood of healthy adults $(n=27)$. Fig. 5. The percentage of BDCA-2+ B7-H4+ dendritic cell in cord blood of healthy neonates $(n=39)$ in comparison with peripheral blood of healthy adults $(n=27)$. 
ty of inducing $\mathrm{T} \mathrm{CD}^{+}$cells is lower than in adults. In addition to this, they are not able to stimulate naive $\mathrm{T}$ cell to IFN- $\gamma$ production [18]. Other authors did not confirm the predominance of immature lymphoid DCs in cord blood of healthy neonates [19].

Dendritic cells play a crucial role in both initiating and down regulating immune response. They are responsible for presenting antigens to naive $\mathrm{T}$ cells, thus initiate as well as regulate immunological response. Their role is important not only in primary responses, but either in immunological tolerance. Recently, Dzionek et al. described three blood dendritic cell antigens: BDCA-1 (CD1c), BDCA-2 (CD303) and BDCA-3 (CD141) which are thought to be markers for human peripheral blood DCs [20]. We can distinguish two subsets of these DCs cells: myeloid den-

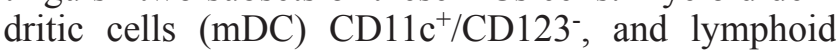
dendritic cells (pDC) CD $123{ }^{\text {brigh }} \mathrm{CD} 11 \mathrm{c}^{-}$. The antigen BDCA-1 is expressed on mDCs, whereas BDCA-2 and BDCA-4 on pDCs cells. There was also described that $\mathrm{mDC}$ produce interleukin-12 (IL-12) and induce Th1, whereas pDCs develop Th2 type response synthesizing interferon (IFN).

To our knowledge, this is the first time study disclosed the difference in the expression of $\mathrm{B} 7-\mathrm{H} 1$ as well as $\mathrm{B} 7-\mathrm{H} 4$ on BDCA- $1^{+}$and BDCA- $-2^{+}$cells in umbilical cord blood in comparison with healthy adults. Our findings shown that both co-stimulatory molecules are highly expressed on either BDCA $-1^{+}$or BDCA $-2^{+}$cells in peripheral blood than in umbilical cord blood. These findings may suggest that low concentration of B7-H1 molecule can induce autoimmune diseases or allergy in neonates. There are findings describing the role of $\mathrm{B} 7-\mathrm{H} 1$ molecule in peripheral blood or in placenta. Activated T cells may be inhibited by this co-stimulator which can bind its receptor called PD-1 [21]. By blocking these receptors it is possible to induce autoimmune disease in organs expressing B7-H1 antigen. In addition to this, they are also detectable on transformed cells as well as in various human cancers. The higher concentration of B7-H1 molecule on DCs in adults underlines its tolerogenic properties. Conversely, binding PD-L1 with PD-1 receptor on activated T-cells and $\mathrm{B}$-cells may inhibit Tcell responses in peripheral tissues by inducing apoptosis and inhibiting cell-cycle progression [1]. All those results proved significant role of B7-H1 molecule in self-tolerance. In addition to this, there is a study where scientists suggest that the expression of $\mathrm{B} 7 \mathrm{H} 1$ on retinal pigment epithelium cells (RPE) plays an inhibitory role via interaction with T cells [22].

For the first time, we either estimated the expression of B7-H4 protein on DCs in umbilical cord blood. We observed higher expressions of this co-stimulatory molecule on both BDCA $-1^{+}$and BDCA- $-2^{+}$dendritic cells in peripheral blood of adults in comparison with umbilical cord blood. The molecule B7-H4 is mainly responsible for an inhibiting proliferation of $\mathrm{CD}^{+}$and $\mathrm{CD}^{+} \mathrm{T}$ cells and depletes cytokine production. It is either capable of inducing programmed cell death. In vitro B7-H4 molecule decreases regulation of T-cells response $[8,9,23,24]$. Kryczek et al. marked that regulatory T-cells (Treg) can induce the expression of B7H4 molecule on different APCs subset via IL-10 production. Moreover, it was described that Tregs cells enable the suppressor activity to APCs via the expression of B7-H4 molecule [25]. Their findings may explain why in our results the expressions of this molecule were higher in peripheral blood of healthy adults.

Some scientists observed the higher expression of B7-H4 molecule on cancer cells. Like c-erbB-2 or p53 molecular indicate the degree of biological aggressiveness of breast cancer [26], the expression of B4-H4 molecule might be clinically useful. The high expression of this molecule may be useful as a marker for detection early stages of cancer [9]. In addition to this Jankowska et al. demonstrated in their study that maturation markers and co-stimulatory molecules on DCspulsed with tumor cell lysate was significantly different in comparison with the expressions in the medium containing only TNF- $\alpha$ [27].

It has been shown that the function of DCs can be characterized by regulation of co-stimulatory molecules (B7.1, B7.2) and HLA-DR. Moreover immature DCs are thought to control peripheral tolerance by inducing the differentiation of human regulatory $\mathrm{T}$ cells $\left(\mathrm{T}_{\text {reg }}\right)$ [28-31]. They are able to induce immune tolerance under certain conditions [32].

In conclusion, dendritic cells may be involved in the immune response of newborns. The decreased percentages of dendritic cells and co-stimulatory molecules indicate that neonates have immature immune system. Although the detailed function of co-stimulatory molecules in cord blood is still largely unknown, the lower expression of immunosuppressive B7-H1 molecule may elucidate its tolerogenic properties in cord blood. Moreover, the depletion of co-stimulatory B7-H1 and B7-H4 molecules enables appropriate development of immune response.

\section{References}

[ 1] Willems F, Vollstedt S, Suter M. Phenotype and function of neonatal DC. Eur J Immunol. 2009;39:26-35.

[2] Banchereau J, Steinman RM. Dendritic cells and the control of immunity. Nature. 1998;392:245-252.

[3] Hart DNJ. Dendritic cells unique leukocyte population which control the primary immune response. Blood. 1997;90:32453287.

[4] Langenkamp A, Messi M, Lanzavecchia A, et al. Kinetics of dendritic cell activation: impact on priming of TH1, TH2 and nonpolarized T cells. Nat Immunol. 2000;1:311-316.

[5] Petroff MG, Kharatyan E, Torry DS, et al. The immunomodulatory proteins $\mathrm{B} 7-\mathrm{DC}, \mathrm{B} 7-\mathrm{H} 2$, and $\mathrm{B} 7-\mathrm{H} 3$ are differentially 
expressed across gestation in the human placenta. Am J Pathol. 2005; 167:465-467.

[ 6] Rudd CE, Schneider H. Unifying concepts in CD28, ICOS and CTLA4 co-receptor signalling. Nat. Rev Immunol. 2003;3:544-556.

[ 7] Watanabe N, Gavrieli M, Sedy JR, et al. BTLA is a lymphocyte inhibitory receptor with similarities to CTLA-4 and PD1. Nat Immunol. 2003;4:670- 679.

[ 8] Kryczek I, Shuang W, Linhua Z, et al. Cutting Edge: Induction of B7-H4 on APCs through IL-10: novel suppressive mode for regulatory T cells. $J$ Immunol. 2006;177:40-44.

[ 9] Kryczek I, Zou L, Rodriguez P, et al. B7-H4 expression identifies a novel suppressive macrophage population in human ovarian carcinoma. $J$ Exp Med. 2006;203:871-881.

[10] Simon I, Zhuo S, Corral L, et al. B7-h4 is a novel membranebound protein and a candidate serum and tissue biomarker for ovarian cancer. Cancer Res. 2006;66:1570-1575.

[11] Liang J, Sun L, Wnag Q, et al. Progesterone regulated mouse dendritic cells differentiation and maturation. Int Immunopharmacol. 2006;5:830-838.

[12] Moser M, De Smedt T, Sornasse T, et al. Glucocorticoids down-regulate dendritic cell function in vitro and in vivo. Eur J Immunol. 1995;25:2818-2824.

[13] Xing N, Maldonado ML, Bachman LA, et al. Distinctive dendritic cell modulation by vitamin $\mathrm{D}(3)$ and glucocorticoid pathways. Biochem Biophys Res Commun. 2002;297:645-652.

[14] Reissland P, Wandinger KP: Increased cortisol levels in human umbilical cord blood inhibit interferon alpha production of neonates. Immunobiology 1999;200:227-233.

[15] Kalinski P, Hilkens CM, Snijders A, et al. Dendritic cells, obtained from peripheral blood precursors in the presence of PGE2, promote Th2 response. Adv Exp Med Biol. 1997; 417:363-367.

[16] Rutella S, Danese S, Leone G. Tolerogenic dendritic cells: Cytokine modulation comes of age. Blood 2006;108:14351440.

[17] Szabolcs P, Park KD, Reese M, Marti L, Broadwater G, Kurtzberg J. Absolute values of dendritic cell subsets in bone marrow, cord blood, and peripheral blood enumerated by a novel method. Stem Cells. 2003;21:296-303.

[18] Willems F, Vollstedt S, Suter M. Phenotype and function of neonatal DC. Eur J Immunol. 2009;39:26-35.

[19] Darmochwal-Kolarz D, Rolinski J, Buczkowski J, Tabarkiewicz J, Leszczynska-Gorzelak B, Zych I, Oleszczuk J. $\mathrm{CD} \mathrm{C}^{+}$immature myeloid dendritic cells are predominant in cord blood of health neonates. Immunology Letters 2004; 91:71-74.
[20] Dzionek A, Fuchs A, Schmidt P et al. BDCA-2, BDCA-3 and BDCA-4: three markers for distinct subsetrs of dendritic cells in human peripheral blood. J Immunol. 2000;165:6037-6046.

[21] Ishida Y, Agata Y, Shibahara K, et al. Induced expression of PD-1, a novel member of the immunoglobulin gene superfamily, upon programmed cell death. EMBO J. 1992;11: 3887-3895.

[22] Usui Y, Okunuki Y, Hattori T et al. Functional expression of B7H1 on retinal pigment epithelial cells. Exp Eye Res. 2008; 86:52-59.

[23] Krambeck AE, Thompson RH, Dong H, Lohse CM, et. al. B7$\mathrm{H} 4$ expression in renal cell carcinoma and tumor vasculature: associations with cancer progression and survival. Proc Natl Acad Sci U S A. 2006; 103:10391-10396.

[24] Mao YX, Chen YJ, Ge Y, et al. Recombinant human B7-H4 expressed in Escherichia coli inhibits T lymphocyte proliferation and IL-2 secretion in vitro. Acta Pharmacol Sin. 2006; 27:741-246.

[25] Kryczek I, Wei S, Zou L, et al. Cutting edge: induction of B7-H4 on APCs through IL-10: novel suppressive mode for regulatory T cells. J Immunol. 2006;177:40-44.

[26] Agrawal AK, Jeleń M, Rudnicki J, Grzebieniak Z, Zukrowski P, Nienartowicz E. Molecular markers (c-erbB-2, p53) in breast cancer. Folia Histochem Cytobiol. 2008;46:449-455.

[27] Jankowska O, Krawczyk P, Wojas-Krawczyk K, et al. Phenotype of dendritic cells generated in the presence of non-small cell lung cancer antigens - preliminary report. Folia Histochem Cytobiol. 2008; 46:465-470.

[28] McLellan AD, Starling GC, Williams LA, et al. Activation of human peripheral blood dendritic cells induces the CD86 costimulatory molecule. Eur J Immunol. 1995;25:2064-2068.

[29] Nijman HW, Kleijmeer MJ, Ossevoort MA, et al. Antigen capture and major histocompatibility class II compartments of freshly isolated and cultured human blood dendritic cells. $J$ Exp Med. 1995;182:163-174.

[30] Dhodapkar MV, Steinman RM, Krasovsky J, et al. Antigenspecific inhibition of effector T cell function in humans after injection of immature dendritic cells. J Exp Med. 2001;193: 233-238

[31] Mahnke K, Qian Y, Knop J, et al. Induction of CD4+/CD25+ regulatory $\mathrm{T}$ cells by targeting of antigens to immature dendritic cells. Blood. 2003;101:4862-4869.

[32] Steinman RM, Hawiger D, Nussenzweig MC. Tolerogenic dendritic cells. Ann Rev Immunol. 2003;21:685-711.

Submitted: 16, December, 2009 Accepted after reviews: 11 July, 2010 